\section{Prospective, Randomized Trial of Doppler-Assisted Subclavian Vein Catheterization}

\author{
RJ Bold, DJ Winchester, AR Madary, et al \\ Departments of Surgical Oncology and Biomathematics, \\ University of Texas, M.D. Anderson Cancer Center, \\ Houston
}

\begin{abstract}
Objective: To examine the rate of success and complications of Doppler-guided subclavian vein catheter insertion compared with standard insertion in patients considered at high risk for failure. Design: Prospective, randomized, crossover trial. Setting: University-affiliated tertiary care medical center. Patients: Two hundred forty patients were enrolled in the study. Patients were stratified for 3 known risk factors: (1) prior surgery in the subclavian vein region, (2) prior radiotherapy at the attempted catheterization site, and (3) an abnormal weightheight ratio. Interventions: Subclavian vein catheterization was performed either in standard or Doppler-guided fashion using the Smart Needle (Peripheral Systems Group, Mountain View, Calif), which is a Doppler probe at the tip of a cannulating needle. If subclavian vein catheterization was unsuccessful after 2 attempts, patients were crossed over to the other technique. Main Outcome Measure: Successful cannulation of the subclavian vein. Results: The success rate, either as an initial technique or as a salvage technique and complication rate were not significantly different with use of the Smart Needle. A subgroup of physicians had a significantly lower success rate using the Smart Needle. Conclusions: Doppler guidance did not increase the success rate or decrease the complication rate of subclavian vein catheterization when compared with the standard technique in high-risk patients. Doppler guidance was not more useful than the standard technique as a salvage technique following a previous failure of catheterization. Furthermore, real-time Doppler guidance of subclavian vein catheterization is a technique that is highly operator dependent. (Arch Surg 133:1089-1093, 1998)
\end{abstract}

COMMENT: This is a large, prospective, randomized, crossover clinical trial from a single institution using a new product that was reported to enhance cannulation of the subclavian vein in routine, elective catheter placement. The device is an 18-gauge needle fitted with a real-time Doppler sensor. It provides information regarding its proximity to vascular structures and may "permit the differentiation of venous and arterial signals." In this well-done study, there was no benefit to the Doppler device.

The authors comment on a previous meta-analysis that suggested opposite findings. The operators in the singleinstitution study were experienced fellows (postgraduate years 6 to 10) using a dedicated procedure suite for the insertion of long-term subclavian venous access catheters. The present study seems to highlight the differences between a single prospective randomized trial and a meta-analysis. The referred meta-analysis pooled eight studies done primarily in critically ill and general medi- cal-surgical patients. In all but two studies, the internal jugular vein was the access site; most operators were junior residents, but two studies used anesthesiologists.

The comparison between the single-center trial and the meta-analysis may represent an "oranges to apples" phenomenon. The Doppler-assisted device for locating venous access may represent a new technology in search of an appropriate application.

Lynne M. Murphy, MSN, RN, CNSN

\section{Efficacy of Antiseptic-Impregnated Central Venous Catheters in Preventing Catheter-Related Bloodstream Infection: A Meta-Analysis}

\author{
DL Veenstra, S Saint, S Saha, et al \\ Department of Pharmacy, University of Washington, \\ Seattle
}

ABSTRACT: Context: Central venous catheters impregnated with chlorhexidine and silver sulfadiazine have recently been introduced for the prevention of catheter-related infections. However, there remains some uncertainty regarding the efficacy of these catheters because of conflicting reports in the literature. Objective: To evaluate the efficacy of chlorhexidine-silver sulfadiazine-impregnated central venous catheters in the prevention of catheter-related bloodstream infection. Data Sources: Studies identified from a computerized search of the MEDLINE database from January 1966 to January 1998, reference lists of identified articles, and queries of principal investigators and the catheter manufacturer. Study Selection: Randomized trials comparing chlorhexidine-silver sulfadiazine-impregnated central venous catheters with nonimpregnated catheters were included. The outcomes assessed were catheter colonization and catheter-related bloodstream infection confirmed by catheter culture. Data Extraction: Twelve studies met the inclusion criteria for catheter colonization and included a total of 2611 catheters. Eleven studies with a total of 2603 catheters met the inclusion criteria for catheter-related bloodstream infection. Most patients in these studies were from groups considered to be at high risk for catheter-related infections. Summary statistics were calculated using Mantel-Haenszel methods under a fixed-effects model. Data Synthesis: The summary odds ratio for catheter colonization was 0.44 (95\% confidence interval [CI], 0.36 to $0.54 ; p<.001$ ), indicating a significant decrease in catheter colonization associated with impregnated catheters. The studies examining the outcome of primary interest; catheter-related bloodstream infection, had a summary odds ratio of $0.56(95 \% \mathrm{Cl}, 0.37$ to $0.84 ; p=$ .005). Conclusions: Central venous catheters impregnated with a combination of chlorhexidine and silver sulfadiazine appear to be effective in reducing the iifidence of both catheter colonization and catheter-related bloodstream infection in patients at high risk for catheter-related infections. (JAMIA 281:261-267, 1999)

COMMENT: This meta-analysis was well conducted. However, the conclusion was predictable. In each of the 11 
trials, the bloodstream infection rate was at least arithmetically lower in the group using the impregnated catheter. If there were truly no difference between the two catheters, the probability of this happening by chance is the same as that of having a coin come up all heads or all tails after 11 tosses, namely <.001. Unless the cost of this catheter is extraordinarily high, there appears to be ample evidence to justify its routine use.

The authors did not hand-search individual medical journals in their effort to find all the pertinent articles, as this process is very time consuming. Sometime in the next century, such a database will be routinely available. The Cochrane Collaboration, an organization dedicated to the production of quality systematic reviews, is currently hand-searching all medical journals. Anyone interested in learning a more about the systematic review process is invited to join them.

Ronald L. Koretz, MD

\section{A Comparison of Two Antimicrobial- Impregnated Central Venous Catheters}

\author{
RO Darouiche, II Raad, SO Heard, et al \\ Department of Medicine, Baylor College of Medicine and \\ Veterans Affairs Medical Center, Houston
}

\begin{abstract}
Background: The use of central venous catheters impregnated with either minocycline and rifampin or chlorhexidine and silver sulfadiazine reduces the rates of catheter colonization and catheter-related bloodstream infection as compared with the use of unimpregnated catheters. We compared the rates of catheter colonization and catheter-related bloodstream infection associated with these two kinds of antiinfective catheters. Methods: We conducted a prospective, randomized clinical trial in 12 university-affiliated hospitals. High-risk adult patients in whom central venous catheters were expected to remain in place for three or more days were randomly assigned to undergo insertion of polyurethane, triple-lumen catheters impregnated with either minocycline and rifampin (on both the luminal and external surfaces) or chlorhexidine and silver sulfadiazine (on only the external surface). After their removal, the tips and subcutaneous segments of the catheters were cultured by both the roll-plate and the sonication methods. Peripheral-blood cultures were obtained if clinically indicated. Results: Of 865 catheters inserted, $738(85 \%)$ produced culture results that could be evaluated. The clinical characteristics of the patients and the risk factors for infection were similar in the two groups. Catheters impregnated with minocycline and rifampin were $1 / 3$ as likely to be colonized as catheters impregnated with chlorhexidine and silver sulfadiazine (28 of 356 catheters [7.9\%] vs 87 of 382 [22.8\%], $p<.001$ ), and catheter-related bloodstream infection was $1 / 12$ as likely in catheters impregnated with minocycline and rifampin ( 1 of 356 [0.3\%], vs 13 of 382 [3.4\%] for those impregnated with chlorhexidine and silver sulfadiazine; $p<.002$ ). Conclusions: The use of central venous catheters impregnated with minocycline and rifampin is associated with a lower rate of infection than the use of catheters impregnated with chlorhexidine and silver sulfadiazine. (N Engl J Med 340:1-8, 1999)
\end{abstract}

COMMENT: It is estimated that 150,000 cases of central venous catheter-related bloodstream infections (CVCBSIs) occur each year in the United States, at a cost of $\$ 30,000$ per case. Current research on the prevention of CVC-BSIs has focused on catheters impregnated with antimicrobials. The minocycline/rifampin (MIR)- and chlorhexidine/silver sulfadiazine (C/SS)-coated catheters are currently available, and each has been shown to reduce the rate of CVC colonization and CVC-BSI when compared with uncoated catheters.

Darouiche and colleagues performed a well-designed study that compared M/R- with C/SS-impregnated CVCs in patients requiring venous access for more than 3 days via a new venipuncture site. The M/R-CVC had a significantly lower rate of colonization than the C/SS-CVC: $7.9 \%$ vs $22.8 \%$, respectively. This protective effect was seen regardless of the amount of time the catheters remained in place. CVC-BSIs occurred in 14 patients, with all but one occurring in the C/SS-CVC group. The rate of CVCBSI was 0.3 vs 4.1 per 1000 catheter days for M/R- and C/SS-CVCs, respectively. The MIR-CVCs were significantly protective against CVC-BSI because of coagulasenegative staphylococci.

This study suggests that $M / R-C V C$ can reduce the rate of infectious complications associated with temporary CVC when compared with C/SS-CVC and that this should result in a decrease in the morbidity, mortality, and cost associated with managing these complications. This conclusion, however, must be made with a few words of caution. First, the rate of catheter colonization and CVCBSI for the C/SS-CVC seen in this study was higher than that reported by others (Maki DG, et al: Ann Intern Med 127:257-266, 1997). Next, although the randomization scheme was appropriate and blinding was maintained in the packaging of the two catheters, it must be noted that the two catheters are different in appearance. This may have introduced a bias in the management of one catheter over another because two of the authors developed the M/R.CVC and the manufacturer of this catheter sponsored the study. Finally, although the use of these catheters has not resulted in the development of bacterial antimicrobial resistance, this issue remains an important concern. Having said this, the results of this and other studies suggest that antibiotic-impregnated catheters may improve the overall care of patients. Hospitals that choose to use these catheters should monitor them closely to assure that their use results in the desired outcomes and that untoward effects are not seen.

Deborah Katz-Scott, RN, CRNI Douglas L. Seidner, MD, CNSP, FACG

\section{A Reevaluation of the Radiographically Detectable Complications of Percutaneous Venous Access Lines Inserted by Four Subcutaneous Approaches}

\author{
JA Miller, S Singireddy, P Maldjian, et al \\ Department of Radiology, The University of Medicine \\ and Dentistry of New Jersey, Newark
}

\begin{abstract}
As a result of prior studies elucidating the potential complications associated with the use of central venous access lines, the Food and Drug Administration and the manufacturers themselves have published guidelines and warnings outlining these dangers and describing the safest insertion techniques. We will attempt to determine whether this improved education has altered the number and type of complications, comparing the results from different types of hospitals, among the various medical services and among operators with varying degrees of experience. This is a prospective analysis of all central venous
\end{abstract}


pressure (CVP) and Swanz-Ganz catheters (SGCs) inserted between July 1, 1995, and February 30, 1996, at a regional Veteran's Affairs hospital and an inner city university medical center. Three hundred seventy-five inpatients underwent 417 new percutaneous venous catheter placements while in the medical or surgical intensive care units or in the general care wards. A portable chest radiograph was obtained immediately after each procedure, and the position of the catheter and any associated complications were recorded, initially by the radiology resident in the emergency suite and subsequently as confirmed by a chest radiology attending. CVP lines were considered malpositioned when the tip was not located in the superior vena cava, and SGCs were recorded as misplaced when the tip was not found within the main, left main, right main, or either lower lobe pulmonary artery within $2 \mathrm{~cm}$ of the cardiac border. For patients undergoing CVP line placement, the right atrium was the most frequent site of tip malposition with 38 of 69 total misplacements (55\%), whereas for SGCs, 22 of 55 malplacements $(40 \%)$ were too distal in a lower lobe pulmonary artery. Only 1 of 248 CVP lines resulted in a pneumothorax (PTX; 0.4\%), whereas 6 of 169 SGC insertions caused a PTX (3.5\%), a significant difference $(p=.019)$. Five of 192 catheters $(2.6 \%)$ placed via an internal jugular approach resulted in PTX, whereas only 2 of 150 subclavian cannulations $(1.3 \%)$ caused this complication, revealing a trend, but not reaching statistical significance $(p=.473)$. However, there was a significant decrease in the rate of catheter misplacements in the third part of the month compared with the first 20 days (35\% vs $24 \% ; p=.031$ ), and the Veteran's Affairs hospital displayed a trend toward more complications for SGC insertions, than the university hospital (47\% vs $32 \%$ ), but not for CVP lines. In general, individual medical and surgical services displayed a similar frequency of complications ( $29 \%$ to $38 \%$ ), except for CVP lines inserted in the medical intensive care unit. Venous access catheter tip malpositions are very common in all settings, but easily recognized by radiography, whereas PTXs are unusual. In contrast to most older studies, PTXs are more frequently observed with internal jugular as opposed to subclavian cannulations and with SGCs rather than CVP lines. However, our data support prior studies that the right atrium and distal right lower lobe pulmonary artery are the most common sites for CVP and SGC misplacement, respectively, and that there is an improvement in success rates with increasing operator experience. (Amer Surg 65:125-130, 1999)
COMMENT: A prospective analysis of 169 Swan-Ganz catheter (SGC) and 248 central venous pressure (CVP) catheter insertions was undertaken to determine if improved education and practitioner experience affected rates of insertion-related complications. Previous studies have shown that subclavian vein insertions have the highest rate of pneumothorax. However, results from this study found a higher rate of pneumothorax from the cannulation of internal jugular veins $(2.6 \%$ vs $1.3 \%$, although not statistically significant). The authors' proposed explanation for this discrepancy was based on the assumption that catheter manufacturers and schools of medicine now have extensive educational materials available regarding the proper technique for subclavian vein cannulation, thereby improving an inexperienced clinician's knowledge and minimizing the risk of complications associated with that approach.

In this study, a statistically significant greater rate of pneumothorax was found with SGC insertions compared with CVP insertions (3.6\% vs $0.4 \% ; p=.019)$. This finding has not been previously reported in the literature. The authors attributed this to the more emergent nature of a SGC insertion and proposed the universal use of cordis sheath introducers to minimize this complication.

Other findings revealed that $67 \%$ of right internal jugular vein insertions resulted with catheter tip malposition in the right atrium and that the rate of catheter malposition was significantly less in the last third of a month compared with the first 20 days (35\% vs $24 \% ; p=$ .031 ), thus, inferring that clinician experience does impact the rate of insertion-related complications. It is interesting to note that the authors' rate of life-threatening complications dramatically improved from the findings published in the literature $10-20$ years ago, again adding credence to their assertion that improved techniques and education positively affected outcomes. Finally, regarding the high rate of catheter malposition, the problem was readily identified in the postinsertion radiographs and could be remedied with catheter retraction into the appropriate vessel.

Susan T. Jepsen, RN, CNSN 